\title{
The use of photographic exposure meters as photometers
}

STANLEY COREN and JOEL MILLER

New School for Social Research, New York, New York 10011

Photographic exposure meters may be used to take reasonably accurate photometric measurements for laboratory purposes if corrections for deviations of the photosensitive elements from the spectral response of CIE standard observer are made. A series of nomographs which directly convert exposure meter readings to $\log$ foot Lamberts are provided for the two most commonly available types of meter. These nomographs incorporate the required corrections for both source and cell characteristics.

One of the most expensive and delicate pieces of equipment found in many behavioral laboratories is the photometer. Such a piece of equipment is critical for the psychophysicist or specialist in visual perception and hence is easily worth the typical expense of more than a thousand dollars, or, at a minimum, several hundred dollars for visual nulling photometers. For the researcher working in learning, clinical, or reading research, the expense of a good photometer seems prohibitive, especially if it is only used once or twice during any experiment. Thus, in these areas it is not infrequent to find stimulus specifications as crude as "the discrimination was established between a bright and a dim light stimulus," or "a dim level of ambient light was available in the test chamber." or "a comfortable level of illumination was present." despite the fact that such important behavioral functions including reaction time, discriminability, and visual acuity, to name but a few, all vary as a function of the levels of illumination (cf. Graham, 1965; or Kling \& Riggs, 1971). The lack of availability of good photometric measuring instruments makes itself particularly evident in field studies or student research. There is, however, a simple effective solution, namely, the cheap, readily available, photographic exposure meter, which may easily be used for the measurement and specification of light intensity.

There are two common types of photographic exposure meters. The simplest involves a selenium (Se) photovoltaic cell which generates an electrical current proportional to the amount of light incident on its surface. The incident light intensity is then displayed in the deflection of a sensitive ammeter attached to the cell. The sensitivity of the meter is a function of both the amount of surface area available to the impinging light and the quality of the meter. Simplicity of construction makes this form of exposure meter quite cheap; many models are available for under $\$ 10$. Another type of exposure meter uses a cadmium-sulfide (Cds) photoconductive element. This element changes resistance as a function of the amount of light reaching its surface. Since it does not generate current, an external power supply is required, usually in the form of a mercury battery. This battery trickles current through the CdS element, and the amount of current flow is registered on a meter. Changes in light intensity vary the resistance, which is reflected in variations in meter readings. With power supplied from a battery, the current levels are such that we can read lower light levels with the same meter movement by employing a $\mathrm{CdS}$ element than with a Se cell. The photoreceptive element can be quite small and still get adequate reactivity. $\mathrm{CdS}$ meters are the meters most frequently employed in behind-the-lens metering systems. In the absence of
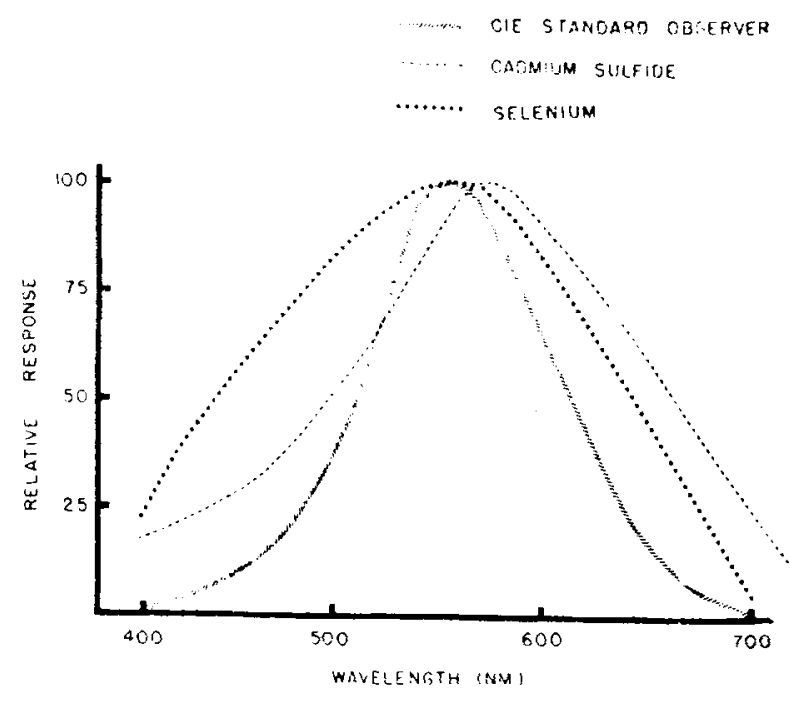

$A$



B

Fig. 1. (A) The relative responses of the CIE standard observer, a typical selenium photovoltaic cell, and a cadmium-sulfide photoconductive element to variations in spectral composition of incident illumination. (B) Relative spectral composition of a daylight fluorescent tube and a $100-\mathrm{W}$ tungsten bulb. 
other information. note that the Se requires no battery and the $\mathrm{CdS}$ does.

It has long been known that the eye is differentially responsive to various wavelengths (i.e., Gibson \& Tyndall, 1923). Its spectral sensitivity has been agreed upon by convention to follow the CIE curve of relative response (CIE. 1924). This curve is the solid line in Fig. 1A. To provide accurate photometric readings, the light sensitive elements in any measuring device should show the same relative response curve found in the CIE standard observer. Precision light measuring devices accomplish this either by incorporating a human eye in the system (i.e.. the Macbeth illuminometer or the SEI exposure photometer) or by incorporating a series of corrective filters in front of the photosensitive element (i.e., Gamma Scientific phiôtumeter). Photographic exposure meters, on the other hand, do not have an exactly correct spectral response. Although Se and $\mathrm{CdS}$ have spectral response curves which approximate that of the eye, peaking in the center of the visible range, there are systematic deviations of their spectral response from the standard observer. Figure $1 \mathrm{~A}$ illustrates that $\mathrm{Se}$ is too sensitive for shorter wavelengths (blue) while $\mathrm{CdS}$ is too sensitive at long wavelengths (red) and that both have broader response bands than the eye. These deviations are not so great as to introduce massive errors in the photometric measurements, because most common light emission sources have relatively broad spectral outputs. However, if there is a predominance of either long or short wavelengths in the spectral output from a light source, readings taken with an uncorrected cell will probably be somewhat in error. There are significant differences in the energy spectra of the two most prevalent light sources, incandescent and fluorescent.

As is shown in Fig. 1B, a general service 100-W incandescent light bulb produces a large percentage of long wavelength light; a daylight fluorescent bulb produces a considerably higher output in the short wave region and actually achieves a correlated color temperature close to the $6500^{\circ} \mathrm{K}$ of mean daylight.

The importance of these spectral differences is that an exposure meter which uses an Se element will tend to underestimate slightly the amount of light present in an incandescent source and a CdS meter will tend to overestimate it. The converse will hold for a fluorescent source: the Se produces an overestimation and the CdS meter an underestimation of the amount of light flux present. Thus, in order to obtain accurate photometric readings, we must know the nature of our photosensitive element and of our light source.

Any attempt to use a photographic exposure meter as a photometer must correct for the spectral response of the cell and for the spectral distribution of the light source. For our purposes, this means establishing the response characteristics of the Se and CdS meters to both incandescent and fluorescent illumination. The Se cell characteristics were obtained from Walsh (1958), combined with manufacturer's specifications from



Fig. 2. Nomographs for conversion of exposure meter readings to $\log \mathrm{fL}$ in incandescent illumination with meter set at ASA 100. (A) For selenium meters; (B) for cadmium-sulfide meters. (See text for complete instructions for use.)

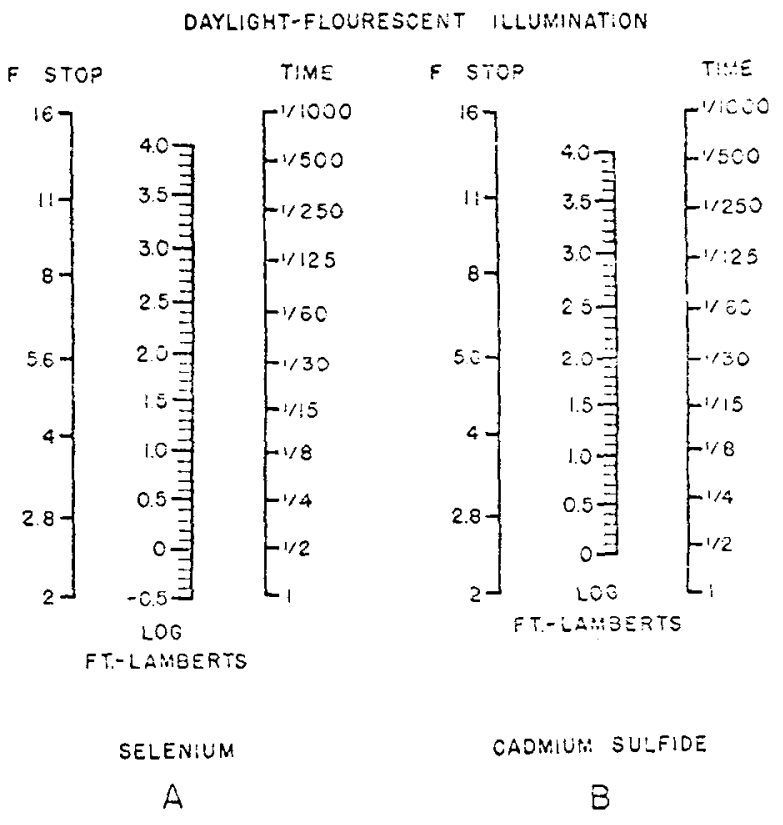

Fig. 3. Nomographs for conversion of exposure meter readings to $\log \mathrm{fL}$ in fluorescent illumination with meter set at ASA 100. (A) For selenium meters; (B) for cadmium-sulfide meters. (See text for complete instructions for use.)

International Rectifier Corporation (1960). Typical response characteristics of $\mathrm{CdS}$ cells were obtained using manufacturer's specifications from RCA (1963) and International Rectifier Corporation (1960). Typical output characteristics of $100-\mathrm{W}$ general service 
incandescent bulbs and daylight fluorescent bulbs were obtained from IES (1966). On the basis of these data, a set of nomographs was prepared which allows the conversion of a photographic exposure reading directily to $\log$ foot Lamberts.

To determine the light intensity of a stimulus, set the ASA indicator on the exposure meter to 100 (or DIN 21). Bring the meter close to the region to be measured. This is necessary to obtain an accurate reading since most exposure meters read an average luminance across the field. If you have a behind-the-lens metering system mounted on a camera, simply fill the viewfinder with the target to be measured. If you have a spot meter, be sure it is centered on the target. Now determine the exposure as accurately as possible from the $F$ stop and shutter speed markings. Since the additon of one F stop doubles the amount of light (i.e., adds $0.3 \log \mathrm{fL}$ ), it is necessary to interpolate between marked values. Thus a value between $F_{2}$ and $F_{2} .8$ is F2.4 if it bisects the interval. To determine the intensity of light coming trom the target stimulus, one need only select the nomograph most appropriate for the light source and meter being used. For incandescent sources, use Fig. 2; for fluorescent (or daylight), use Fig. 3. Now select the graph appropriate for your meter and use a straight edge to connect the obtained $F$ stop with the obtained exposure time. The point where the straight edge intersects the $\log \mathrm{fL}$ scale indicates the intensity of the target. Thus, if you use a selenium meter to measure the brightness from a piece of white paper illuminated by an incandescent light and obtain an exposure of $F 8$ and $1 / 60$, lay a straight edge between $F 8$ and $1 / 60$ on Fig. 2A and read $2.20 \mathrm{log} \mathrm{fL}$. If you wish to increase sensitivity to lower illuminations, simply set the ASA on the exposure meter to $1000($ DIN $=31)$ and reduce all readings by one log unit. In the example above, if the ASA were 1000 , you would read $1.2 \log \mathrm{fL}$. To increase the upward range of the instrument so that readings on brighter sources may be obtained, simply reduce the ASA to $10(\mathrm{DIN}=11)$ and add one $\log$ unit to the reading (for the example given, we would read $3.2 \mathrm{log}$ fL). For most purposes, however, the range provided by an initial setting of ASA 100 on the exposure meter should suffice.

In order to ascertain the accuracy of the photometric measures, a series of test trials were undertaken. All measures were verified against a Gamma Scientific photometer which incorporates a photomultiplier tube, a filter corrected to match the CIE standard observer's response characteristics, and is calibrated against a standardized light source of $100 \mathrm{fL}$. Two test light sources were used, a $2900^{\circ} \mathrm{K} 100-\mathrm{W}$ tungsten lamp, whose intensity was controlled by passing the light through an iconel neutral density wedge. and a daylight fluorescent lamp whose intensity was similarly controlled by a neutral density wedge. Two selenium exposure meters. one a Gossen Scout and the other a Wesson, and two cadmium-sulfide meters, one camera mounted on a Yashica Penta J5 and the other a behind-the-lens meter in a Nikormat, were used to estimate the readings taken with a calibrated photometer. A range of stimulus intensities from $3 \mathrm{log}$ $\mathrm{fL}$ to $0.2 \log \mathrm{fL}$ were employed; four readings were taken at each of 14 points spaced at approximately $0.2 \log \mathrm{fL}$ intervals. The mean percentage error for the exposure meter readings was $7.2 \%$ as compared with the photometer. Thus, it seems reasonable to conclude that the photographic exposure meter used in conjunction with the nomographs provides direct measures of light intensity within an acceptable range of accuracy for most purposes. If increased accuracy is desired, or if the light being tested is a broad band source which differs significantly in spectral composition from the specific tungsten or the fluorescent sources used in the construction of the nomographs, a simple correction factor can be obtained. This requires access either to a photometer or to a light source of known intensity, for only one measurement. The intensity of the source is measured using the exposure meter, and the reading in $\log$ foot Lamberts is obtained from the nomograph most appropriate to the photocell and source used. Suppose that the test source is known to be $2 \log \mathrm{fL}$ and the obtained reading is $2.3 \log \mathrm{fL}$ from the graph. This means that, for the photocell source combination you are using, your readings will be too high by a constant factor of $0.3 \log \mathrm{fL}$ for the entire range. This correction can now be applied to all future readings using that particular exposure meter for similar sources. In this hypothetical instance, one need only subtract 0.3 from all readings. Thus, a value of $1 \log f \mathrm{~L}$ corresponds to $0.7 \log \mathrm{fL}$. Such a correction procedure greatly improves the accuracy of the readings and increases the variety of sources which may be measured. It also allows reflected light readings from surfaces that night not be specifically neutral. Using such correction and repeating the calibration measures taken above, the mean percent error for the sources and exposure meters tested is reduced to a value of $2.3 \%$.

It is clear that the photographic exposure meter is not a substitute for accurate photometric measurements in the areas of visual perception and sensory physiology where the utmost precision in specification of light intensities is required. It is also unlikely that narrow band light sources, such as those obtained from a monochrometer or through highly selective filters, can be read with any reasonable accuracy given the deviations of the spectral response of the photoelements in exposure meters from the CIE standard observer (although when corrected as above. the accuracy of readings becomes reasonable). Another limitation is the difficulty in interpolating between $F$ stop or shutter values. In spite of limitations, the photographic exposure meter seems to be an adequate and inexpensive instrument which will meet most student and many laboratory needs. It can be used to ascertain the luminance level in a tachistoscope, the light incident on 
a stimulus card in a display box, the luminance level present in an animal training cage, or in other experimental situations where measurements of light intensity are desirable from a broad spectral source.

\section{REFERENCES}

CIE Proceedings 1924, Cambridge, England: University Press, 1926.

Gibson, K. S., \& Tyndall, E. P. T. Visibility of radiant energy U.S. Bureau of Standards Scientific Paper No. 475, 1923, 19 131-191.
Graham, C. H. (Ed.) Vision and visual perception. New York: Wiley, 1965.

IES lighting handbook. New York: Illumination Engineering Society, 1965

International Rectifier Corporation. Solar cell and photo cell handbook. E1 Sugundo, Calif.: International Rectifier Corporation, 1960.

Kling, J. W., \& Riggs, L. A. (Eds.) Woodworth and Schlosberg's experimental psychology. New York: Holt, Rinehart, \& Winston, 1971 .

R.C.A. Phototubes and photocells, Lancaster, $\mathrm{Pa}$.: Radio Corporation of America, 1963.

Waish, J. W. T. Photometry. New York: Dover, 1958.

(Received for publication February 21, 1973 ; revision received April 30,1973 ). 